$06,11,13$

\title{
Об аномальном влиянии внешнего электрического поля на фазовый переход второго рода в тонкой сегнетоэлектрической пленке
}

\author{
(C) B.Н. Нечаев, А.В. Шуба \\ Военный учебно-научный центр Военно-воздушных сил \\ „Военно-воздушная академия им. профессора Н.Е. Жуковского и Ю.А. Гагарина“, \\ Воронеж, Россия \\ E-mail: shandvit@rambler.ru
}

Поступила в Редакцию 27 августа 2019 г.

В окончательной редакции 12 декабря 2019 г.

Принята к публикации 25 ноября 2019 г.

В рамках феноменологической теории Ландау-Гинзбурга-Девоншира исследованы особенности фазового перехода в тонкой сегнетоэлектрической пленке во внешнем электрическом поле в зависимости от толщины пленки и типа закрепления поляризации на ее поверхности. Обнаружено, что в электрическом поле изменяется механизм фазового перехода. Помимо специфического размытия, отличающегося от размытия в объемном материле появлением вблизи $T_{\mathrm{C}}$ температурного интервала с аномально высокой диэлектрической восприимчивостью, фазовый переход смещается по температуре, причем величина смещения зависит от толщины пленки, свойств ее поверхности и в слабых полях не зависит от напряженности поля.

Ключевые слова: фазовый переход, сегнетоэлектрическая пленка, электрический потенциал, внешнее электрическое поле.

DOI: 10.21883/FTT.2020.04.49122.574

\section{1. Введение}

Как известно [1], в объемном образце при фазовом переходе (ФП) второго рода структура материала изменяется непрерывно, в то время как симметрия его в точке перехода меняется скачком. Такое поведение материала приводит к скачку теплоемкости, обращению в нуль обратной восприимчивости и аномалиям других свойств в точке $T_{\mathrm{C}}$ ФП. Приложенное внешнее поле, сопряженное параметру порядка, например, электрическое в случае сегнетоэлектриков, индуцирует соответствующее изменение симметрии материала, и точка ФП, как точка скачкообразного изменения симметрии, исчезает. Вследствие этого на температурной шкале пропадает точка сингулярности термодинамических характеристик материала и они становятся гладкими функциями температуры - внешнее поле „размывает“ ФП второго рода.

Такая достаточно простая ситуация не сохраняется в случае образцов конечных размеров и, в частности, тонких сегнетоэлектрических пленок (СЭП), представляющих модельный объект исследования в настоящей статье. ФП в этой ситуации происходит по механизму потери устойчивости и описывается как появление нетривиальных решений у соответствующей краевой задачи на собственные значения [2,3]. Несмотря на смену механизма $Ф П$, температура $T_{f}$-перехода как точка сингулярности термодинамических характеристик при этом сохраняется, но смещается, как правило, вниз по температурной шкале [3,4]. Меняется также и механизм влияния внешнего поля на ФП - краевая задача становится неоднородной и существование нетривиальных решений, т.е. разрешимость ее, определяется альтернативой Фредгольма [5]. В итоге изменяется как температура ФП - включение внешнего поля приводит к ее конечному сдвигу, - так и вид решения вблизи точки ФП.

\section{2. Математическая модель и решение краевой задачи}

Целью настоящей работы является определение смещения температуры ФП и температурного интервала размытия перехода при приложении внешнего электрического поля в зависимости от толщины СЭП и типа закрепления поляризации на ее поверхности с учетом собственных деполяризующих полей.

Рассмотрим тонкую СЭП толщиной $l$ с вектором поляризации $\mathbf{P}=\{0,0, P\}$, перпендикулярным плоскости $(x, y)$ пленки, претерпевающую ФП второго рода. Свободную энергию такой пленки вблизи точки ФП второго рода запишем согласно теории Ландау-Гинзбурга-Девоншира [6,7]:

$$
\begin{aligned}
F= & \int_{V}\left(-\frac{\alpha}{2} \mathbf{P}^{2}+\frac{\beta}{4} \mathbf{P}^{4}+\frac{\kappa}{2}(\nabla P)^{2}-\mathbf{E P}\right) d V \\
& -\int_{V_{\infty}} \frac{\varepsilon}{8 \pi} \mathbf{E}^{2} d V+\int_{S} \frac{\alpha_{s}}{2} \mathbf{P}^{2} d S,
\end{aligned}
$$

где $\alpha=\alpha_{0}\left(T_{\mathrm{C}}-T\right), \beta$ и $\alpha_{s}-$ соответственно коэффициенты разложения свободной и поверхностной энергий в ряд по степеням параметра порядка $P-$ поляризации, 
до второго и четвертого порядка, соответственно, вблизи температуры ФП $T_{\mathrm{C}}[2] ; \kappa \approx \alpha_{0}^{2}-$ корреляционная постоянная, $a_{0}$ - параметр кристаллической решетки; $\mathbf{E}$ - вектор напряженности электрического поля, включающий как деполяризующее, так и внешнее поле, $\varepsilon-$ вклад в диэлектрическую проницаемость (преимущественно электронный), не учитываемый с помощью параметра порядка. В первом интеграле выражения (1) интегрирование ведется по объему $V$ СЭП, во втором по всему пространству, окружающему пленку, включая и ее саму, в третьем - по поверхности $S$-пленки.

Варьирование функционала (1) по поляризации $P$ и электрическому потенциалу $\varphi$ дает искомые уравнения равновесия и граничные условия к ним, определяющие ФП в тонкой СЭП.

Структурные ФП, не сопровождающиеся возникновением дальнодействующих электрических полей, представляют собой частный случай описываемой модели. Рассмотрим сначала именно эту наиболее простую ситуацию для иллюстрации влияния внешнего поля на ФП.

\section{1. Структурный фазовый переход}

Пусть $P=\eta$ - параметр порядка, описывающий атомные смещения при ФП, не приводящие к возникновению электрических полей, $h-$ сопряженное ему внешнее поле. Уравнение равновесия и граничные условия в этом случае имеют вид

$$
\begin{gathered}
\kappa \frac{d^{2} \eta}{d z^{2}}+\alpha \eta-\beta \eta^{3}=-h, \\
\left.\kappa \frac{d \eta}{d z} \mp \alpha_{s} \eta\right|_{z=0, l}=0 .
\end{gathered}
$$

Для проведения расчетов в целях удобства перейдем к безразмерным переменным: $\tilde{z}=z / a_{0}, \tilde{l}=l / a_{0}$, $\tilde{\alpha}=\alpha_{s} / a_{0}, \quad \tilde{\eta}(z)=\eta(z) / \eta_{0}, \quad \tilde{h}=h / \eta_{0}$, где $\eta_{0}-$ спонтанное значение параметра порядка объемного 3Dкристалла. В дальнейшем, работая с безразмерными переменными для упрощения записи тильду в обозначениях будем опускать. Кроме того, для определения только температуры ФП можно опустить нелинейное слагаемое в уравнении (2). Тогда система (2), (3) в новых переменных примет вид

$$
\begin{gathered}
\frac{d^{2} \eta}{d z^{2}}+\alpha \eta=-h, \\
\left.\frac{d \eta}{d z} \mp \alpha_{s} \eta\right|_{z=0, l}=0 .
\end{gathered}
$$

В отсутствие внешнего поля $(h=0)$ ФП в пленке описывается однородной системой (4), (5), и в настоящее время является достаточно хорошо изученным $[2,3,8]$ в разных материалах. Согласно $[2,3,8]$, температура $T_{f} \Phi П$ определяется как температура появления ненулевого решения этой системы. Поскольку однородная система соотношений (4), (5) с математической точки зрения представляет собой задачу Штурма-Лиувилля, то температура ФП будет определяться первым собственным значением этой задачи. Для его нахождения общее решение однородного уравнения (4)

$$
\eta(z)=C_{1} \cos \sqrt{\alpha} z+C_{2} \sin \sqrt{\alpha} z
$$

подставляем в граничные условия (5) и получаем однородную систему линейных уравнений для нахождения констант интегрирования $C_{1}, C_{2}$ :

$$
\left\{\begin{array}{l}
\alpha_{s} C_{1}-\sqrt{\alpha} C_{2}=0 \\
\left(\alpha_{s} \cos \sqrt{\alpha} l-\sqrt{\alpha} \sin \sqrt{\alpha} l\right) C_{1} \\
\quad+\left(\alpha_{s} \sin \sqrt{\alpha} l+\sqrt{\alpha} \cos \sqrt{\alpha} l\right) C_{2}=0
\end{array}\right.
$$

Условие равенства нулю определителя системы (7) дает трансцендентное уравнение

$$
\left(\alpha_{s}^{2}-\alpha\right) \sin \sqrt{\alpha} l+2 \alpha_{s} \sqrt{\alpha} \cos \sqrt{\alpha} l=0,
$$

определяющее температуру $T_{f}$ ФП в СЭП. Уравнение (8) анализировалось в работе [9]. Согласно [9], в зависимости от $\alpha_{s}$ значение $T_{f}$ будет отвечать какойлибо точке интервала $\left(T_{\mathrm{C}}-\frac{\pi^{2}}{\alpha_{0} l^{2}}, T_{\mathrm{C}}\right)$. Нижняя его граница соответствует случаю $\alpha_{s} \rightarrow \infty$, верхняя $-\alpha_{s}=0$.

При включении внешнего поля функция $\eta(z)$ будет представлять собой решение неоднородной задачи (4), (5), которое находится методом вариации произвольных постоянных

$$
\begin{aligned}
\eta(z)= & C_{1} \cos \sqrt{\alpha} z+C_{2} \sin \sqrt{\alpha} z \\
& +\frac{\cos \sqrt{\alpha} z}{\sqrt{\alpha}} \int h(z) \sin \sqrt{\alpha} z d z \\
& -\frac{\sin \sqrt{\alpha} z}{\sqrt{\alpha}} \int h(z) \cos \sqrt{\alpha} z d z .
\end{aligned}
$$

Здесь и ниже, считая внешнее поле однородным $(h(z)=h)$ и подставляя функцию (9) в граничные условия (5), получаем неоднородную систему линейных алгебраических уравнений для нахождения констант интегрирования $C_{1}, C_{2}$

$$
\left\{\begin{array}{l}
\alpha_{s} C_{1}-\sqrt{\alpha} C_{2}=\frac{\alpha_{s} h}{\alpha}, \\
\left(\sqrt{\alpha} \sin \sqrt{\alpha} l-\alpha_{s} \cos \sqrt{\alpha} l\right) C_{1} \\
\quad-\left(\sqrt{\alpha} \cos \sqrt{\alpha} l+\alpha_{s} \sin \sqrt{\alpha} l\right) C_{2}=-\frac{\alpha_{s} h}{\alpha} .
\end{array}\right.
$$

Детерминант матрицы коэффициентов системы (10) совпадает с левой частью уравнения (8) и при температуре $T=T_{f}$ обращается в нуль. Согласно теореме Кронекера-Капелли, чтобы система (10) при $T=T_{f}$ имела решение, необходимо, чтобы ранг расширенной матрицы системы, так же как и ранг матрицы коэффициентов, был бы равен единице. Это возможно, когда все 
определители второго порядка расширенной матрицы равны нулю

$$
\left\{\begin{array}{l}
\sin \sqrt{\alpha} l=-\frac{2 \alpha_{s} \sqrt{\alpha}}{\alpha_{s}^{2}+\alpha} \\
\cos \sqrt{\alpha} l=\frac{\alpha_{s}^{2}-\alpha}{\alpha_{s}^{2}+\alpha}
\end{array}\right.
$$

Отметим, что фактически использованный здесь метод представляет собой частный случай более общего результата - альтернативы Фредгольма, примененной к неоднородной алгебраической системе уравнений. Можно заметить, что множество решений уравнения (8) включает в себя множество решений системы (11). Пересечение этих множеств определяет температуру ФП пленки во внешнем поле. Численный анализ системы (8), (11) представлен на рис. 1, где показана зависимость температуры $T_{f}^{h}$ ФП в поле от приведенных толщины пленки $l$ и параметра $\alpha_{s}$. При построении графиков использовались параметры кристалла триглицинсульфата (ТГС): $T_{\mathrm{C}}=322 \mathrm{~K}, \alpha_{0}=3.92 \cdot 10^{-3} \mathrm{~K}^{-1}$, $\varepsilon=30[10]$.

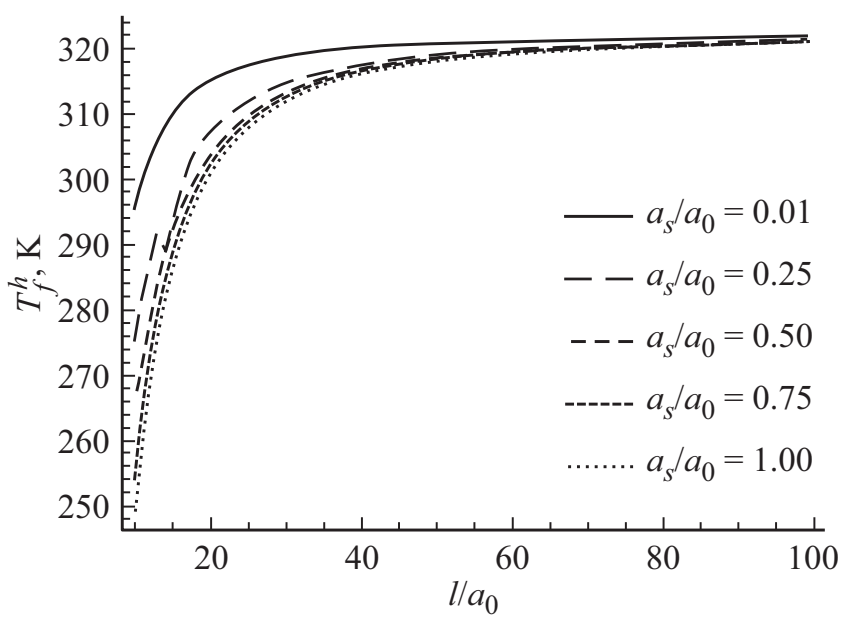

Рис. 1. Зависимость температуры $T_{f}^{h}$ ФП от толщины пленки во внешнем поле.

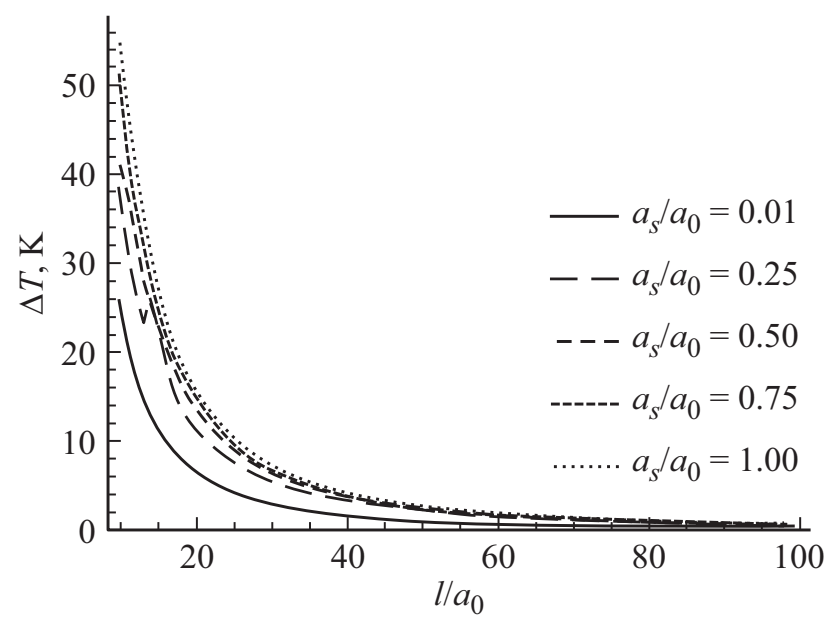

Рис. 2. Зависимость смещения $\Delta T$ температуры ФП от толщины пленки во внешнем поле.
Предельные случаи допускают аналитическое рассмотрение. Если параметр порядка не закреплен на границе пленки $\left(\alpha_{s}=0\right)$, то система уравнений $(8),(11)$ принимает вид

и имеет решение

$$
\left\{\begin{array}{l}
\sin \sqrt{\alpha} l=0 \\
\cos \sqrt{\alpha} l=-1
\end{array}\right.
$$

$$
T_{f}^{h}=T_{\mathrm{C}}-\frac{\pi^{2}}{\alpha_{0} l^{2}} .
$$

В другом предельном случае $\left(\alpha_{s} \rightarrow \infty\right)$ система уравнений $(8),(11)$ принимает вид

$$
\left\{\begin{array}{l}
\sin \sqrt{\alpha} l=0 \\
\cos \sqrt{\alpha} l=1
\end{array}\right.
$$

и имеет решение

$$
T_{f}^{h}=T_{\mathrm{C}}-\frac{4 \pi^{2}}{\alpha_{0} l^{2}} .
$$

Значение $T_{f}^{h}$ зависит от величины $\alpha_{s}$ и принадлежит интервалу

$$
\left(T_{\mathrm{C}}-\frac{4 \pi^{2}}{\alpha_{0} l^{2}}, \quad T_{\mathrm{C}}-\frac{\pi^{2}}{\alpha_{0} l^{2}}\right) .
$$

На рис. 2 приведена зависимость смещения $\Delta T=T_{f}-T_{f}^{h} \quad$ температуры $Ф П$ от толщины СЭП во внешнем поле.

\section{2. Сегнетоэлектрический фазовый переход}

Полученные выше результаты качественно применимы для тонких СЭП. Однако для корректного сравнения теории с экспериментом необходимо учитывать деполяризующие электрические поля, сопровождающие неоднородное распределение поляризации в СЭП и оказывающие существенное влияние на ФП в ней $[2,11]$. Учитывая связь электрического потенциала $\varphi$ и напряженности электрического поля $\mathbf{E}$

$$
\mathbf{E}=-\nabla \varphi,
$$

и варьируя функционал (1) по поляризации $P$ и электрическому потенциалу $\varphi$, получим систему уравнений, описывающую ФП в этом случае:

а) для области внутри пленки $(0<z<l)$

$$
\left\{\begin{array}{l}
\frac{d^{2} P}{d z^{2}}+\alpha P-\frac{d \varphi_{i}}{d z}=0 \\
\frac{d^{2} \varphi_{i}}{d z^{2}}-\frac{4 \pi}{\varepsilon} \frac{d P}{d z}=0
\end{array}\right.
$$

б) для областей вне пленки $z<0$ или $z>l$, обозначенных индексами 1 и 2 ,

$$
\varepsilon_{e} \frac{d^{2} \varphi_{e}^{(i)}}{d z^{2}}=0, \quad i=1,2,
$$

где $\varphi_{i}, \varphi_{e}$ - электрический потенциал, соответственно, внутри и вне пленки; $\varepsilon_{e}$ - диэлектрическая проницаемость окружения. При записи уравнений (12), (13), 
как и ранее, использовались безразмерные переменные, причем электрический потенциал $\varphi$ был нормирован на величину $P_{0} a_{0}$. Общее решение для уравнений $(12),(13)$ имеет вид

$$
\begin{gathered}
P(z)=C_{1} \cos \sqrt{\tilde{\alpha}} z+C_{2} \sin \sqrt{\tilde{\alpha}} z+C_{3}, \\
\varphi_{i}(z)=\frac{4 \pi}{\varepsilon \sqrt{\tilde{\alpha}}} C_{1} \cos \sqrt{\tilde{\alpha}} z-\frac{4 \pi}{\varepsilon \sqrt{\tilde{\alpha}}} C_{2} \sin \sqrt{\tilde{\alpha}} z+\alpha z C_{3}+C_{4},
\end{gathered}
$$

$$
\begin{aligned}
& \varphi_{e}^{(1)}(z)=-h z+C_{5}, \\
& \varphi_{e}^{(2)}(z)=-h z+C_{6},
\end{aligned}
$$

где $h-$ внешнее электрическое поле, в котором находится СЭП, $\tilde{\alpha}=\alpha-4 \pi / \varepsilon$. Так как электрический потенциал $\varphi_{i}(z)$ можно определять с точностью до константы, то величину $C_{4}$ можно положить равной нулю. Граничные условия для уравнений (12), (13) в тех же безразмерных переменных имеют вид

$$
\left\{\begin{array}{l}
\left.\frac{d P}{d z} \mp \alpha_{s} P\right|_{z=0, l}=0, \\
\left.\varphi_{i}\right|_{z=0}=\left.\varphi_{e}^{(1)}\right|_{z=0}, \\
\left.\varphi_{i}\right|_{z=l}=\left.\varphi_{e}^{(2)}\right|_{z=l}, \\
\frac{d \varphi_{i}}{d z}-\left.\frac{4 \pi}{\varepsilon} P\right|_{z=0}=-\left.\frac{\varepsilon_{e}}{\varepsilon} h\right|_{z=0} .
\end{array}\right.
$$

Подстановка функций (14), (15) в граничные условия (16) образует неоднородную систему линейных уравнений относительно констант интегрирования $C_{1}$, $C_{2}, C_{3}, C_{5}, C_{6}$ :

$$
\left\{\begin{array}{l}
\alpha_{s} C_{1}-\sqrt{\tilde{\alpha}} C_{2}+\alpha_{s} C_{3}=0 \\
\left(\alpha_{s} \cos \sqrt{\tilde{\alpha}} l-\sqrt{\tilde{\alpha}} \sin \sqrt{\tilde{\alpha}} l\right) C_{1} \\
\quad+\left(\alpha_{s} \sin \sqrt{\tilde{\alpha}} l+\sqrt{\tilde{\alpha}} \cos \sqrt{\tilde{\alpha}} l\right) C_{2}+\alpha_{s} C_{3}=0 \\
\frac{4 \pi}{\varepsilon \sqrt{\tilde{\alpha}}} C_{2}+C_{5}=0 \\
\frac{4 \pi}{\varepsilon \sqrt{\tilde{\alpha}}} \sin \sqrt{\tilde{\alpha}} l C_{1}-\frac{4 \pi}{\varepsilon \sqrt{\tilde{\alpha}}} \cos \sqrt{\tilde{\alpha}} l C_{2}+\alpha l C_{3}-C_{6}=-h l \\
\tilde{\alpha} C_{3}=-\frac{\varepsilon_{e}}{\varepsilon} h .
\end{array}\right.
$$

При отсутствии внешнего электрического поля $(h=0)$ равенство нулю детерминанта системы (17) дает трансцендентное уравнение для нахождения нетривиального решения:

$$
\left(\alpha_{s}^{2}-\tilde{\alpha}\right) \sin \sqrt{\tilde{\alpha}} l+2 \alpha_{s} \sqrt{\tilde{\alpha}} \cos \sqrt{\tilde{\alpha}} l=0,
$$

которое отличается от уравнения (8), определяющего температуру $T_{f}$ ФП без деполяризующих полей, заменой $\tilde{\alpha}=\alpha$. Другими словами, при учете собственных электрических полей температура ФП в СЭП смещается на величину $4 \pi /\left(\varepsilon \alpha_{0}\right)$. Для определения температуры $T_{f}^{h}$ ФП во внешнем электрическом поле исследуем систему (17) на совместность. Поскольку ранг ее матрицы коэффициентов равен четырем, то, по теореме Кронекера-Капелли для разрешимости системы необходимо, чтобы все определители расширенной матрицы четвертого порядка тоже были равны нулю. Это требование приводит к системе из четырех уравнений:

$$
\left\{\begin{array}{l}
\alpha_{s} \sin \sqrt{\tilde{\alpha}} l+\sqrt{\tilde{\alpha}}(1+\cos \sqrt{\tilde{\alpha}} l)=0, \\
\sqrt{\tilde{\alpha}} \sin \sqrt{\tilde{\alpha}} l+\alpha_{s}(1-\cos \sqrt{\tilde{\alpha}} l)=0, \\
\frac{4 \pi \alpha_{s}}{\varepsilon \sqrt{\tilde{\alpha}}}\left[\sqrt{\tilde{\alpha}} \sin \sqrt{\tilde{\alpha}} l+\alpha_{s}(1-\cos \sqrt{\tilde{\alpha}} l)\right] \\
\quad-\alpha l\left[\left(\alpha_{s}^{2}-\tilde{\alpha}\right) \sin \sqrt{\tilde{\alpha}} l+2 \alpha_{s} \sqrt{\tilde{\alpha}} \cos \sqrt{\tilde{\alpha}} l\right]=0 \\
\left(\alpha_{s}^{2}-\tilde{\alpha}\right) \sin \sqrt{\tilde{\alpha}} l+2 \alpha_{s} \sqrt{\tilde{\alpha}} \cos \sqrt{\tilde{\alpha}} l=0 .
\end{array}\right.
$$

Третье уравнение системы (19) есть линейная комбинация второго и четвертого, поэтому его можно опустить. Последнее уравнение системы (19) совпадает с уравнением (18) для нахождения температуры $T_{f}$ ФП. Первое и второе уравнение системы (19) формально совпадают с уравнениями системы (11) для определения температуры $T_{f}^{h}$ ФП в несегнетоэлектрической пленке во внешнем поле, если положить $\tilde{\alpha}=\alpha$.

Таким образом, собственные электрические поля понижают температуру ФП в СЭП относительно температуры $T_{f}$ структурного ФП, а также температуру ФП в СЭП во внешнем электрическом поле относительно температуры $T_{f}^{h}$ в несегнетоэлектрической пленке на постоянную величину $4 \pi /\left(\varepsilon \alpha_{0}\right)$, не зависящую от толщины пленки и свойств ее поверхности. Тогда зависимость температуры $T_{f}^{h}$ ФП от толщины СЭП во внешнем электрическом поле будет отличаться от рис. 1 только сдвигом всех кривых как целого вдоль оси ординат вниз на величину $4 \pi /\left(\varepsilon \alpha_{0}\right) \approx 107 \mathrm{~K}$, а смещение $\Delta T=T_{f}-T_{f}^{h}$ температуры ФП будет полностью соответствовать рис. 2 .

\section{3. Короткозамкнутый сегнетоэлектрический конденсатор}

Особенность данной ситуации заключается в том, что электрические заряды на поверхности пленки будут нейтрализованы зарядами электродов, и поля рассеяния вне пленки не возникают, но внутри нее деполяризующие поля будут существенно неоднородными по толщине. Напряженность внешнего электрического поля, в котором находится СЭП, имеет вид

$$
h=-\frac{\varphi_{i}(l)-\varphi_{i}(0)}{l},
$$

откуда потенциал на верхней плоскости пленки $\varphi_{i}(l)=-h l+\varphi_{i}(0)$. Положив для удобства расчетов 
потенциал на нижней плоскости равным нулю, получим граничные условия

$$
\left\{\begin{array}{l}
\left.\frac{d P}{d z} \mp \alpha_{s} P\right|_{z=0, l}=0, \\
\varphi_{i}(0)=0 \\
\varphi_{i}(l)=-h l
\end{array}\right.
$$

Подстановка функций (14) в граничные условия (20) приводит к системе линейных уравнений относительно констант интегрирования $C_{1}, C_{2}, C_{3}, C_{4}$ :

$$
\left\{\begin{array}{l}
\alpha_{s} C_{1}-\sqrt{\tilde{\alpha}} C_{2}+\alpha_{s} C_{3}=0 \\
\left(\alpha_{s} \cos \sqrt{\tilde{\alpha}} l-\sqrt{\tilde{\alpha}} \sin \sqrt{\tilde{\alpha}} l\right) C_{1} \\
\quad+\left(\alpha_{s} \sin \sqrt{\tilde{\alpha}} l+\sqrt{\tilde{\alpha}} \cos \sqrt{\tilde{\alpha}} l\right) C_{2}+\alpha_{s} C_{3}=0 \\
\frac{4 \pi}{\varepsilon \sqrt{\tilde{\alpha}}} C_{2}-C_{4}=0 \\
\frac{4 \pi}{\varepsilon \sqrt{\tilde{\alpha}}} C_{1} \sin \sqrt{\tilde{\alpha}} l-\frac{4 \pi}{\varepsilon \sqrt{\tilde{\alpha}}} C_{2} \cos \sqrt{\tilde{\alpha}} l+\alpha C_{3} l-C_{4}=-h l
\end{array}\right.
$$

При отсутствии внешнего поля условие равенства нулю детерминанта системы $(21)$

$$
\begin{aligned}
\frac{8 \pi \alpha_{s}}{\varepsilon \sqrt{\tilde{\alpha}}} & {\left[\sqrt{\tilde{\alpha}} \sin \sqrt{\tilde{\alpha}} l+\alpha_{s}(1-\cos \sqrt{\tilde{\alpha}} l)\right] } \\
& -\alpha l\left[\left(\alpha_{s}^{2}-\tilde{\alpha}\right) \sin \sqrt{\tilde{\alpha}} l+2 \alpha_{s} \sqrt{\tilde{\alpha}} \cos \sqrt{\tilde{\alpha}} l\right]=0
\end{aligned}
$$

дает трансцендентное уравнение определения температуры $T_{f}$ ФП. Температура $T_{f}^{h}$ ФП при замыкании цепи конденсатора находится из условия разрешимости неоднородной системы (21) - условия равенства нулю всех определителей четвертого порядка расширенной матрицы. Решая полученную систему уравнений, определяем смещение $\Delta T$. Результаты численных расчетов представлены на рис. 3 .

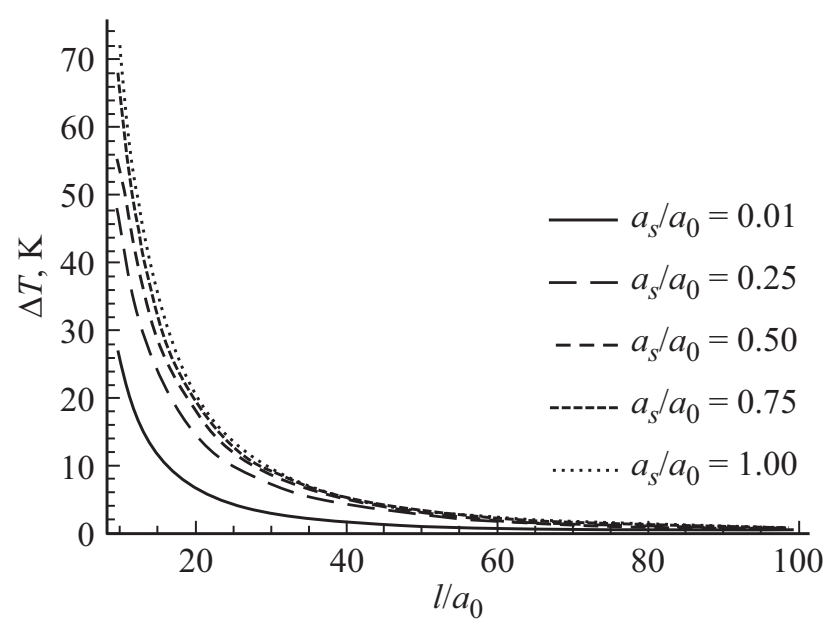

Рис. 3. Зависимость смещения $\Delta T$ температуры ФП от толщины СЭП в электрическом поле конденсатора.
Из рис. 3 видно, что с ростом параметра $\alpha_{s}$, пропорционального степени закрепления поляризации на поверхности СЭП, смещение $\Delta T$ температуры ФП увеличивается сильнее, чем в предыдущем случае (рис. 2), что связано с неоднородными электрическими полями внутри пленки.

Уширение $\varepsilon$ в тонких СЭП экспериментально наблюдалось в работах $[12,13]$, но связывалось это с проявлением флексоэлектрического эффекта.

Смещение температуры ФП во внешнем поле не зависит от величины этого поля только в достаточно слабых полях. Зависимость от внешнего поля в данной ситуации проявляется через зависимость области применимости используемой математической модели от внешнего поля, конкретно, линейного приближения. Условие применимости линейного приближения можно представить в виде неравенства

$$
\max _{z \in[0, l]}\left|\beta P^{3}(z)\right| \ll h,
$$

которое, с учетом (9), приводится к виду

$$
h \ll \frac{\pi^{3}}{2 l^{3} \sqrt{2 \beta}} .
$$

Для пленки ТГС $\left(\beta=8 \cdot 10^{-10}\right.$ ед. СГСЭ) условие (24) определяет напряженность внешнего поля $h \ll 0.39$ ед. СГСЭ $=116 \mathrm{~V} / \mathrm{cm}$, выше которой полученные здесь результаты относительно независимости смещения температуры ФП от напряженности приложенного поля не будут иметь силу.

\section{3. Заключение}

В настоящей работе описаны новые представления о механизме ФП в тонкой СЭП под действием внешнего электрического поля, предполагающие помимо размытия ФП дополнительное смещение его температуры. Величина этого смещения не зависит от напряженности прикладываемого электрического поля (в рамках применимости данной модели), а определяется толщиной пленки, типом материала и параметром поверхности $\alpha_{s}$. Так, смещение $\Delta T$ температуры ФП в поле для пленки ТГС толщиной $l=10 \mathrm{~nm}$ в зависимости от величины параметра $\alpha_{s}$ будет лежать в интервале от 6.3 до $18.9 \mathrm{~K}$.

Точка обращения в нуль обратной диэлектрической восприимчивости $\chi^{-1}(T)=0$ на температурной шкале есть точка ФП во внешнем поле - этот вывод верен для объемного сегнетоэлектрика, но не всегда для пленки, в частности, для ситуации, рассмотренной в работах $[14,15]$. В них авторами неявно предполагалось, что в пленке, как и в объемном сегнетоэлектрике, влияние внешнего поля сводится только к размытию ФП. То, что кроме размытия есть еще дополнительное смещение температуры ФП, связанное с внешним полем, в этих работах не предполагалось и, естественно, не исследовалось. Если же ФП происходит в присутствии внешнего 
поля то при строгом последовательном анализе неоднородных краевых задач (4), (5) и (12), (13), (16) следует учитывать, что при температуре $T_{f}$ для уравнения (4) или (12) появляется ненулевое решение $\left(T_{f}\right.$ отвечает первому собственному значению краевой задачи). Это, в полном соответствии с теорией краевых задач, означает, что решение неоднородной задачи будет существовать не всегда, а только при соблюдении дополнительных условий - соотношений (11), (19). Эти условия выполняются при температуре $T_{f}^{h}$, которая и является температурой ФП в пленке, поскольку в этой точке появляется спонтанная поляризация. Таким образом, во внешнем поле температура ФП смещается на величину $\Delta T=T_{f}-T_{f}^{h}$, которая сильно зависит от толщины исследуемого объекта и принимает заметные значения только в тонких пленках толщиной менее $30 \mathrm{~nm}$.

В температурном интервале $\left(T_{f}, T_{f}^{h}\right)$ неоднородные краевые задачи (4), (5) и (12), (13), (16) не имеют решений. Следовательной, несправедливы допущения, сделанные при их формулировке, в частности, предположение об однородности распределения поляризации и деполяризующих полей в плоскости пленки, позволившее свести задачу к обыкновенным дифференциальным уравнениям. В этом случае следует решать систему уравнений в частных производных, заменяя дифференциальный оператор $\frac{d^{2}}{d z^{2}}$ в формуле (4) на оператор более общего вида $\frac{d^{2}}{d x^{2}}+\frac{d^{2}}{d z^{2}}$. Исходя из опыта решения подобных задач, можно предположить, что в указанном температурном интервале будут существовать неоднородные состояния как, например, в работах [2,16], вероятная причина появления которых связана с включением внешнего поля. Для иллюстрации этой ситуации можно привести, следуя выкладкам работы [17], выражения для поляризации в самом простом случае структурного ФП

$$
\begin{aligned}
P(z)= & h e^{-i q x}\{\sin \sqrt{\hat{\alpha}} l \\
& +\frac{\alpha_{s}}{\sqrt{\hat{\alpha}}}(\cos \sqrt{\hat{\alpha}} z-2 \cos \sqrt{\hat{\alpha}} l+\cos \sqrt{\hat{\alpha}}(l-z)) \\
& \left.+\left(\frac{\alpha_{s}}{\sqrt{\hat{\alpha}}}\right)^{2}(\sin \sqrt{\hat{\alpha}} z-\sin \sqrt{\hat{\alpha}} l+\sin \sqrt{\hat{\alpha}}(l-z))\right\} \\
& \times\left\{\left(\hat{\alpha}-\alpha_{s}^{2}\right) \sin \sqrt{\hat{\alpha}} l-2 \alpha_{s} \sqrt{\hat{\alpha}} \cos \sqrt{\hat{\alpha}} l\right\}^{-1},
\end{aligned}
$$

где $\mathbf{q}\{q, 0,0\}-$ волновый ветор, определяющий характерный размер неоднородного распределения поляризации в плоскости пленок; $\hat{\alpha}=\sqrt{\alpha+q^{2} \kappa}$. В целом же эта задача имеет самостоятельный интерес, а ее исследование выходит за рамки настоящей статьи. Температура $\Phi П$, найденная в работах $[14,15]$ из условия $\chi^{-1}(T)=0$, есть, по существу, температура перехода в неоднородное состояние, если ФП происходит в присутствии внешнего поля.
Резюмируя, отмечаем особенности механизма ФП во внешнем поле: во-первых, происходит дополнительное смещение температуры ФП на величину $\Delta T=T_{f}-T_{f}^{h}$; во-вторых, имеет место размытие ФП. Но размытие ФП в этом случае отличается от размытия в объемном материале. Так, в работе [1] под размытием ФП во внешнем поле понимают монотонное, без скачков, изменение поляризации, диэлектрической восприимчивости и термодинамических характеристик системы при приближении к температуре $T_{f}$. В таких пленках, помимо этого, появляется температурный интервал $\Delta T=T_{f}-T_{f}^{h}$ с аномально высокой диэлектрической восприимчивостью, отвечающий неоднородным состоянием поляризации.

Рассмотренная в статье ситуация представляет еще один пример аномальных физических явлений, когда сколь угодно слабое внешнее воздействие порождает конечный отклик системы. В данной ситуации причина конечного смещения температуры ФП в малом внешнем электрическом поле состоит в том, что поле меняет симметрию среды, в которой находится СЭП, и в ней появляется выделенное направление. Существуют и другие примеры подобных явлений. Малая вязкость качественно меняет течение жидкости, как в пограничном слое, так и во всем окружающем пространстве, создавая завихренность [18]. В результате появляется конечная сила сопротивления, подъемная сила при движении тела в жидкости. Связано это с тем, что вязкость, вопервых, является сингулярным возмущением, поскольку в уравнения гидродинамики добавляются пространственные производные более высокого порядка и меняются граничные условия; во-вторых, вязкость нарушает симметрию - исчезает операция обращения во времени, что и приводит к конечным эффектам даже при исчезающе малой вязкости. В квантовой механике известен эффект образования связанного состояния в неглубокой потенциальной яме, неспособной к локализации частицы без поля, при включении сколь угодно слабого магнитного поля [19]. При его воздействии меняется симметрия среды, в которой происходит движение частицы, и задача становится похожей на движение в одномерной потенциальной яме, где связанные состояния образуются всегда [20]. Известно влияние магнитного поля на пластичность диа- и парамагнетиков. Несмотря на то, что прямое силовое воздействие магнитного поля на магнитные примеси - стопоры для движения дислокаций - несущественно даже в максимально достижимых экспериментально магнитных полях, эффект имеет заметную величину $[21,22]$. Это связывается с тем, что в присутствии магнитного поля открываются новые пути спин-зависимых реакций образования и разрыва ковалентных связей.

Отметим, что обнаруженный в статье эффект, - есть один из возможных путей объяснения размытия ФП в сегнеторелаксорах, вследствие наличия в них неоднородных внутренних электрических полей, обусловленных дефектной структурой [23]. 


\section{Конфликт интересов}

Авторы заявляют, что у них нет конфликта интересов.

\section{Список литературы}

[1] Л.Д. Ландау, Е.М. Лифшиц. Теоретическая физика. Т. 5. Статистическая физика. Ч. 1. Физматлит, М. (2002). 616 с.

[2] В.Н. Нечаев, А.В. Шуба. ФТТ 60, 7, 1322 (2018).

[3] V.N. Nechaev, A.V. Shuba. Ferroelectrics 444, 18 (2013).

[4] В.Н. Нечаев, А.В. Шуба. ФТТ 56, 5, 949 (2014).

[5] И.Г. Петровский. Лекции по теории интегральных уравнений. Наука, М. (1965). 128 с.

[6] К.М. Рабе, Ч.Г. Ан, Ж.-М. Трискон, Б.А. Струков, А.И. Лебедев. Физика сегнетоэлектриков: современный взгляд. Лаб. знаний, М. (2015). 443 с.

[7] Л.Д. Ландау, Е.М. Лифшиц. Теоретическая физика. Т. 8. Электродинамика сплошных сред. Физматлит, М. (2005). $656 \mathrm{c}$.

[8] V.N. Nechaev, A.V. Shuba. Ferroelectrics 359, 35 (2007).

[9] V.N. Nechaev, A.V. Shuba. Ferroelectrics 501, 32 (2016).

[10] Г.А. Смоленский, В.А. Боков, В.А. Исупов, Н.Н. Крайник, Р.Е. Пасынков, М.С. Шур. Сегнетоэлектрики и антисегнетоэлектрики. Наука, Л. (1971). 476 с.

[11] V.N. Nechaev, A.V. Viskovatykh, A.V. Shuba. IOP Conf. Ser.: Mater. Sci. Eng. 525, 012009 (2019).

[12] L.G. Sinnamon, R.M. Bowman, J.M. Gregg. Appl. Phys. Lett. 81, 889 (2002).

[13] G. Catalan, L.G. Sinnamon, J.M. Gregg. J. Phys.: Condens. Matter 16, 2253 (2004).

[14] M.D. Glinchuk, E.A. Eliseev, V.A. Stephanovich. Phisica B 322, 356 (200).

[15] M.D. Glinchuk, A.N. Morozovska, E.A. Eliseev. J. Appl. Phys. 99, 114102 (2006).

[16] I.A. Luk'yanchuk, L. Lahoche, A. Sene. Phys. Rev. Lett. 102, 147601 (2009).

[17] В.Н. Нечаев, А.В. Шуба. Сб. тр. ХІІ междунар. конф. „ПМТУКТ-2019“, ВГУИТ, Воронеж (2019). С. 234.

[18] Л.Д. Ландау, Е.М. Лифшиц. Теоретическая физика. Т. 6. Гидродинамика. Ч. 1. Физматлит, М. (2001). 736 с.

[19] Ю.Н. Демков, Г.Ф. Друкарев. ЖЭТФ 49, 1(7), 257 (1965).

[20] А.И. Базь, Я.Б. Зельдович, А.М. Переломов. Рассеяние, реакции и распады в нерелятивистской квантовой механике. 2-е изд. Наука, М. (1971). 544 с.

[21] Ю.И. Головин, Р.Б. Моргунов. ЖЭТФ 115, 2, 605 (1999).

[22] Ю.И. Головин, Р.Б. Моргунов, В.Е. Иванов, А.А. Дмитриевский. ЖЭТФ 116, 2, 1080 (2000).

[23] С.Б. Вахрушев, С.Г. Жуков, В.В. Чернышев. ФТТ 41, 7, 1282 (1999).

Редактор Е.Ю. Флегонтова 